\title{
MICROGEIGER: A MICROFABRICATED GAS-BASED BETA RADIATION DETECTOR
}

\author{
Chester G. Wilson* and Yogesh B. Gianchandani \\ Department of Electrical Engineering and Computer Science, University of Michigan, Ann Arbor
}

\begin{abstract}
This paper reports a micromachined Geiger counter fabricated from a glass-Si-glass stack of wafers. As a beta particle passes through, a bias applied between two enclosed electrodes generates electron cascades in the gas between them. This results in a current pulse or "count". A single die of $2 \mathrm{~cm}^{2}$ had 6 independent chambers ranging in size from $8 \times 8 \mathrm{~mm}$ to $1 \times 3 \mathrm{~mm}$. $\mathrm{He}, \mathrm{Ne}$, and a He/air mixture, which have different voltage bias requirements, are separately evaluated as background gases. Counting rates are lower in a $\mathrm{Ne}$ background gas than in $\mathrm{He}$, but $\mathrm{Ne}$ is more suitable for packaging. In tests the device was found to detect incident beta particles from a Uranium-238 source. Counting rates of up to 22 counts/min were measured. As with conventional Geiger counters, the rates varied inversely with distance from the source. The microGeiger was tested with pure $\mathrm{He}$ and $\mathrm{Ne}$ background gas with ${ }^{90} \mathrm{Sr}$, ${ }^{60} \mathrm{Co}$, and ${ }^{204} \mathrm{Tl}$, all beta emitting isotopes. Rates up to 24 counts/min. were measured.
\end{abstract}

\section{INTRODUCTION}

Radioactive materials, particularly uranium, are stockpiled in large and small quantities all over the world with varying degrees of security. There is a perpetual risk of their potential use in "dirty bombs," which use conventional explosives to disperse dangerous radioactive materials. Uranium-238 naturally decays into ${ }^{234} \mathrm{Th}$ and then ${ }^{234 \mathrm{~m}} \mathrm{~Pa}$, emitting $0.8 \mathrm{MeV}$ beta particles, which are essentially high energy electrons. Other possible dirty bomb ingredients include a number of beta sources, including ${ }^{90} \mathrm{Sr}$ and ${ }^{204} \mathrm{Tl}$. The former is a particularly hazardous material, as it is easily absorbed into the human body, where it displaces calcium in bone, remaining there with a radioactive half-life of 27 years. Thus, there is considerable motivation to develop miniaturized sensors for radioactive materials.

Since a few radioactive materials emit X-rays, one possibility is to exploit X-ray detectors, which have benefited from solid-state technology in recent years [1]. Unfortunately, most radioisotopes are not sources of Xrays, and the best way to detect most of the target species for dirty bombs is through their emission of beta particles. Solid-state detectors for beta particle exist, but they are relatively large, with sizes on the order of $1 \mathrm{~cm}^{2}$. They typically require cryogenic cooling to distinguish radiation

*Corresponding author: chwilsn@engin.umich.edu. type and energy, and are particularly subject to radiation damage [2]. Another type of device uses pixelated silicon structures at room temperature to provide spatial imaging of beta particle flux [3].

Geiger counters, however, are the preferred sensors for detecting beta radiation [4]. Typical Geiger counters utilize a tube held under vacuum, with a rod-like anode and concentric cathode. (Fig. 1). The tube is biased at 500-1000 volts, and a thin window - typically mica - allows passage of beta radiation. This radiation ionizes the gas at some statistical rate, resulting in an avalanche breakdown, which is measured by circuitry as a "count" corresponding to one event. These gas-based detectors are very reliable, temperature insensitive, require only simple circuitry, and measure over a much wider range of radiation species and energies. Miniaturized gas-based detectors exist, but work only for detection of photon based radiation, such as X-rays [5]. Again, as very few isotopes emit $\mathrm{X}$-rays, there is a compelling need for a micromachined beta radiation detector.

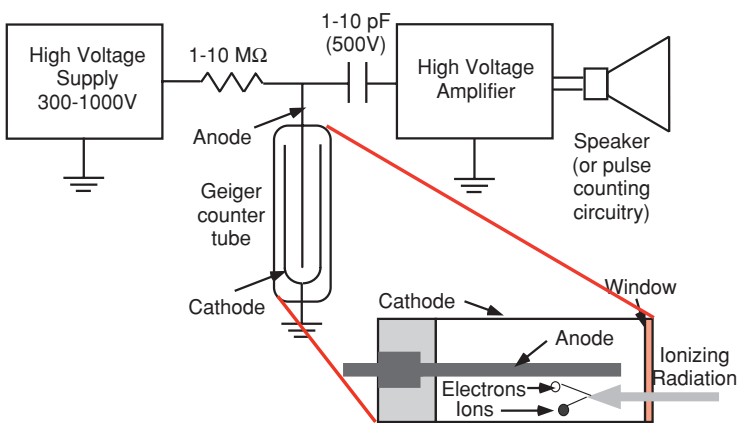

Fig. 1: Traditional Geiger counters utilize a tube under partial vacuum, and a high voltage supply applied through a resistor to a small capacitor.

\section{DEVICE CONCEPTS AND OPERATION}

The microGeiger device is fabricated by anodic bonding a glass-silicon-glass stack, where the silicon is EDP etched to form an anode-cathode configuration. When DC power is applied to this configuration two electrostatic regions are formed: a drift and amplification region (Fig. 2). As beta particles pass through the glass window into the drift region, they ionize the background gas. The electrons are slowly accelerated into the amplification region. In the amplification region the electrons are quickly accelerated 
through a higher field region resulting in an electron cascade. Designing the drift region to be much larger than the amplification region allows consistent pulses that are not a function of the entry position of the beta particle.

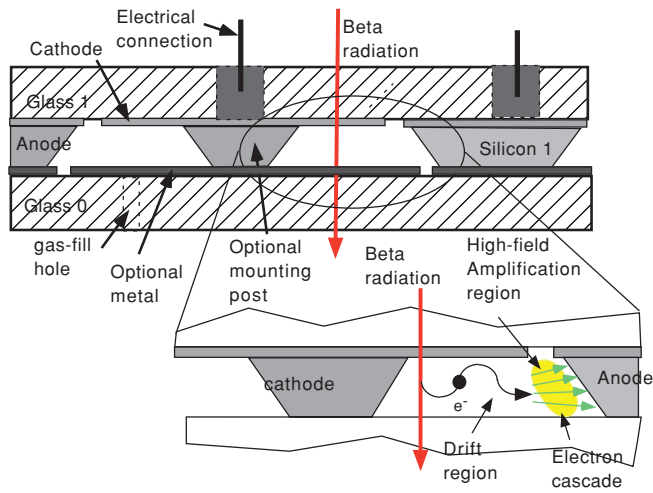

Fig. 2: The microGeiger device utilizes dissolved silicon bonded to glass as the anode-cathode configuration. Beta radiation passing through the drift region creates liberated electrons, which travel to the amplification region, creating an electron cascade.

Macroscale gas-based devices have been widely used in the field of radiation detection [6]. Virtually all gasbased detectors rely on the impinging radiation ionizing the fill gas, with the resulting electrons accelerated by an electric field, ionizing more neutral species, thereby creating an avalanche breakdown. The general form of the electron density in a cascade of length $x$ is given by:

$$
n(x)=n(0) \exp (\alpha x)
$$

Here, $\alpha$ is the first Townsend coefficient of the gas, a function of the gasses ionizability, and electron capture cross section.

Typical detectors fall into four regimes of operation, defined by the applied electric field, electrode geometry, and the pressure and species of fill gas (Fig. 3). The four regimes all have electric discharges with differing physical properties. (These regimes apply to both beta particles and photons like X-rays and gamma particles). The regime with the lowest voltage is the ion saturation region, where the only charge collected is by gas directly ionized by impinging radiation. As the voltage across the device is increased, avalanche breakdown begins to occur, and the amount of collected current increases. This is the proportional region: the amount of current is roughly proportional to the energy of impinging X-rays or gamma particles, as photon radiation is completely absorbed by the background gas. In contrast, impinging beta particles impart only a portion of their kinetic energy to the ionization of gas, so the resulting current created is not correlated to the beta energy; the proportional region is more limited for beta particles.

As the voltage is increased, the dependence of the current pulse upon the energy of the radiation is diminished even for photon radiation. This non-linearity is primarily due to the difference in mobilities between ions and electrons. In the limited proportionality regime, the much slower ions are sufficient in quantity to create a spacecharge region which distorts the local electric fields. This limits the total charge, such as created by avalanching and is dependent on the electric fields. (Similar space-charge regions have been found to be the reason for lower charge densities in previously reported microplasmas [6].) As the voltage is increased further, the impinging radiation generates a self-sustaining discharge; this is the GeigerMuller region. The total amount of current collected in this region for a cylindrical proportional counter is given by:

$$
\ln M=\frac{V}{\ln (b / a)} \cdot \frac{\ln 2}{\Delta V}\left(\ln \frac{V}{p a \ln (b / a)}-\ln K\right)
$$

Here, $M$ is the multiplication factor, the quantity of electrons from a single incident. $V$ is the applied voltage, $a$ and $b$ are the anode and cathode radii, respectively; $p$ is the ambient gas pressure, and $\Delta V$ and $K$ are constants of the background gas, related to electron mean free path and ionizability.

For the characteristic dimensions, and gas species of the microGeiger device, a corresponding cylindrical configuration would provide an $M$, multiplication factor ranging from 10 to $10^{5}$ as the operating voltages are varied. The actual multiplication of the microGeiger device is in the range of $1-2 \times 10^{13}$. This is because the microGeiger device operates in the Geiger-Muller regime, where the initial charge multiplication is further amplified by secondary emission from the anode. This serves as compensation for the small size of the microGeiger, in which the amplification region is smaller than in traditional devices.

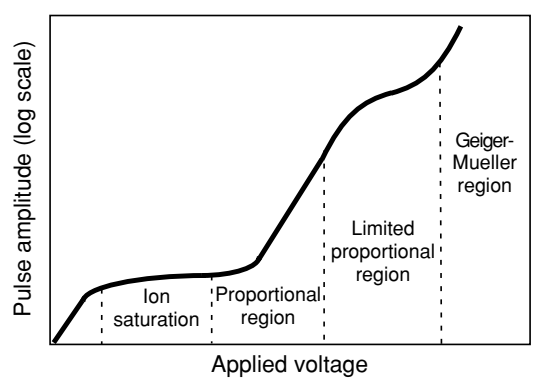

Fig. 3: Gas-based radiation detectors have four regimes of operation, that are defined by the applied voltage.

\section{DEVICE FABRICATION}

The microGeiger device is fabricated in either a two or three mask process (Fig. 4). The first mask defines a borondiffused etch stop. Mask two patterns an oxide which defines a region where silicon is etched. This silicon wafer is anodically bonded to a pyrex wafer, which forms the beta window. The silicon is etched, forming structural offsets, and boron-doped anodes and cathodes. The stack is diced and bonded to a second glass wafer, providing the capability 
of gas packaging. Photographs of the final die with six independent cavities is shown in Fig. 5.

\section{EXPERIMENTAL RESULTS}

Unsealed devices were tested in a flow chamber to permit the comparison of various fill gasses (Fig. 7). The background gas flows into the microGeiger device through input ports machined into the glass window. The gasses which were evaluated included $\mathrm{He}, \mathrm{Ne}$, and a He/Air gas mixture. Helium was chosen for its lower ionization energy; this allows a larger current pulse at microscale dimensions. Neon also has a lower ionization energy, but does not leak through metal. In the test set-up DC power was provided to a capacitor which powers the anode; the cathode was grounded. Figure 8 shows a uranium ore sample that was used to test the devices. For all uranium tests a He/Air gas mixture was used as the background fill gas. Figure 9 illustrates the counting rate of one chamber in the microGeiger as a function of the distance from the detector. The normalized decline in counts is similar to that measured with a conventional detector, an ElectroNeutronics CDV-700.

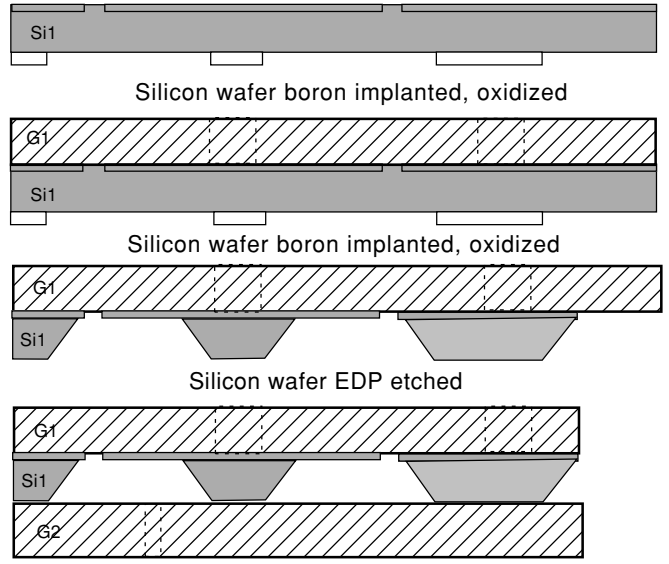

Wafer diced and bonded to glass window

Fig. 4: Process flow of the microGeiger device. Mask 1 defines a boron etch stop, mask 2 a patterned oxide. EDP etching, anodic bonding, and additional glass processing define the microGeiger cavities.

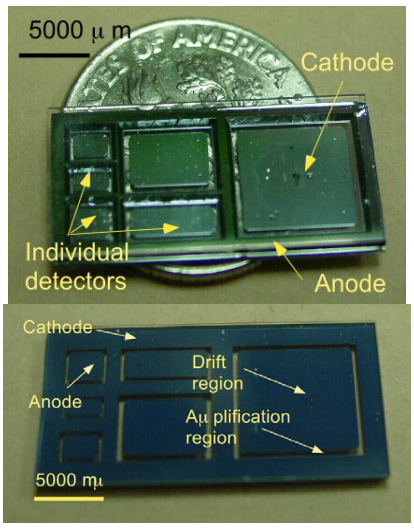

Fig. 5 (a-upper) The microGeiger die contains multiple detector cavities and is orders of magnitude smaller than traditional tubes. (b-lower) Backside of the microGeiger device.
Figure 10 shows the counts per minute as a function of the thickness of the glass windows. As the thickness of the window increases, more beta radiation is absorbed before it reaches the encapsulated gas as the glass thickness is increased. This illustrates that standard glass wafers from $500 \mu \mathrm{m}-750 \mu \mathrm{m}$ can be utilized for fabrication.

As the discharge capacitor that powers the microGeiger device is charged to increasing voltages the charge delivered in each pulse increases, however, the final voltage on the discharge capacitor after ignition remains fairly constant (Fig. 11). This provides evidence that a selfsustaining discharge is created in the device that terminates when the capacitor reaches a certain voltage.

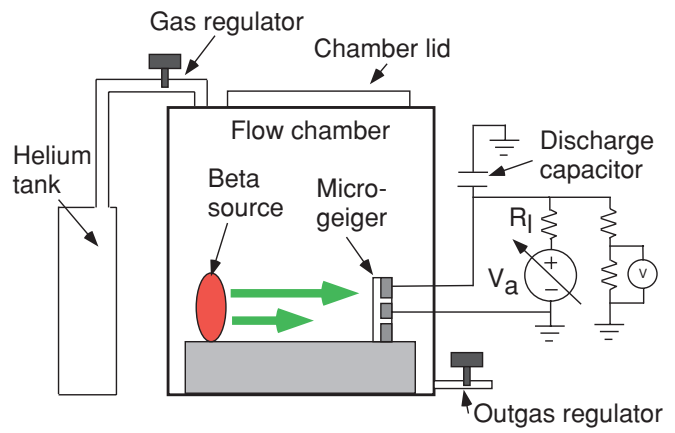

Fig. 7: Preliminary test setup for the microGeiger device; tank allows for helium filling, and pulse discharge measurement. Final microGeiger devices have the gas sealed within them, and do not require the chamber.

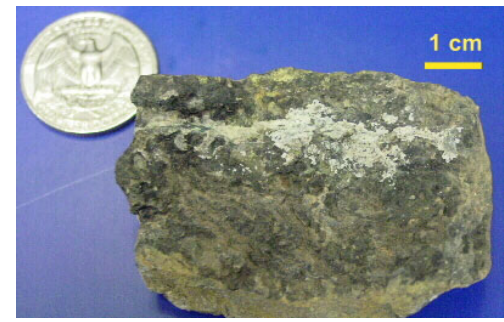

Fig. 8: Uranium ore sample used to test device.

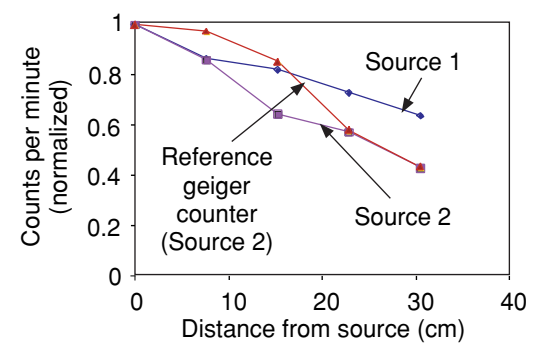

Fig. 9: Normalized counts per minute for the microGeiger and a reference Geiger counter as a function of source distance.

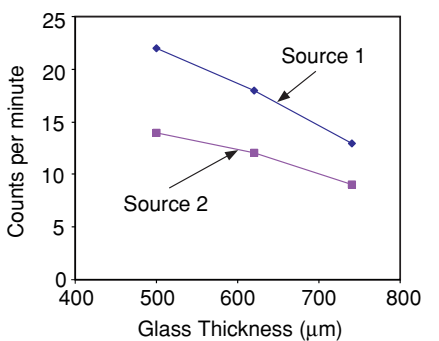

Fig. 10: Over 20 counts per minute are realized on the microGeiger. 200 $\mu \mathrm{m}$ thickness of glass reduces the counting rate by about a factor of 2 . 


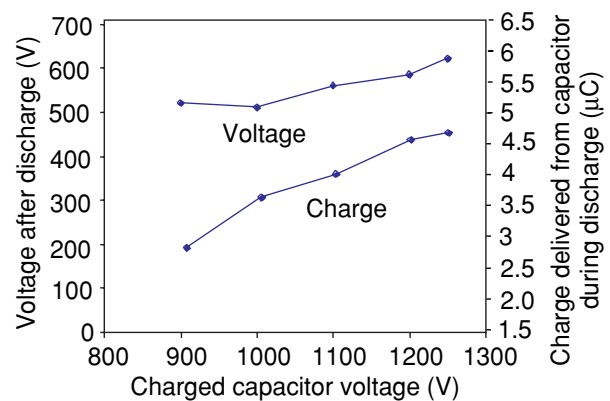

Fig. 11: Increasing the capacitor bias increases the amount of charge delivered to the discharge. The residual voltage on the capacitor remains fairly constant

The microGeiger device was tested with a variety of radioactive isotopes, and fill gases. The corresponding results are shown in Figs. 12-14. Figure 12 illustrates data for a $500 \mu \mathrm{m}$ thick Pyrex window, and He fill gas. Count rates were measured for three different cavity dimensions ( $8 \times 8 \mathrm{~mm}, 4 \times 4.5 \mathrm{~mm}$, and $2 \times 2 \mathrm{~mm}$ ), and two isotopes, ${ }^{90} \mathrm{Sr}$ and ${ }^{60} \mathrm{Co} .{ }^{90} \mathrm{Sr}$ emits $0.546 \mathrm{MeV}$ beta particles, and ${ }^{60} \mathrm{Co}$ provides a range of beta particles, with a maximum energy of $0.314 \mathrm{MeV}$. The count rate can be seen to increase as the cavity size increases, providing more collection area, for both isotopes. Figure 13 provides data for Ne fill gas, using ${ }^{90} \mathrm{Sr}$ and ${ }^{204} \mathrm{Tl}$ isotopes. ${ }^{204} \mathrm{Tl}$ produces a spectrum of beta particles with a maximum energy of $0.776 \mathrm{MeV}$. Again, the counting rate is seen to increase as the collection area increases.

Eventually, as the voltage is increased in the microGeiger device, a continuous arc breakdown occurs across the anode-cathode gap. Pulsed breakdown due to the impinging beta particle occurs at voltages that are lower, but still sufficiently high to develop the avalanche breakdown. This defines the allowed voltage operating range for the device, which is shown for three different background gasses in Fig. 14. Helium provides the lowest operating voltages but can be difficult to package long term, as it leaks through metal. Neon eliminates this problem with only a slight increase in operating voltage. He/Air mixtures provide a much wider range of allowed voltages, however the counting rate becomes a function of the operating voltage.

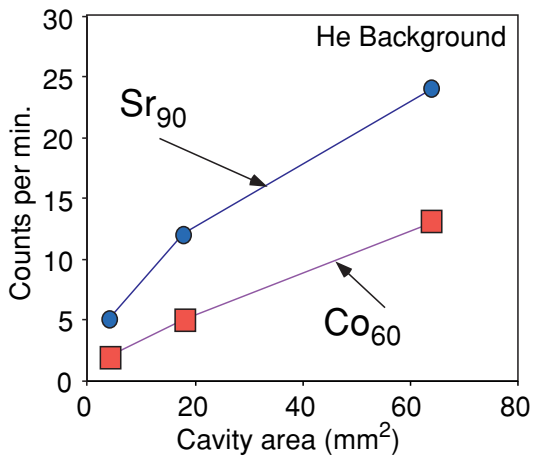

Fig. 12: Counting rates for a $\mathrm{He}$ packaged device, for varying cavity sizes.

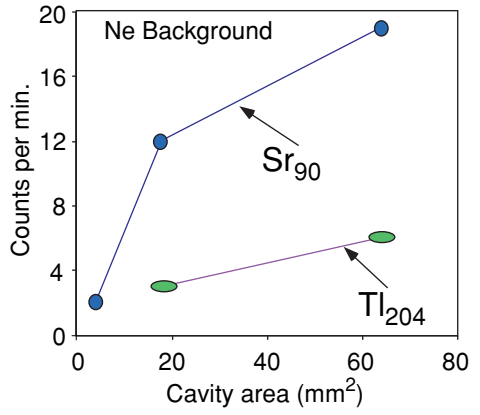

Fig. 13: Counting rates seen in a $\mathrm{Ne}$ packaged device, with varying cavity sizes.

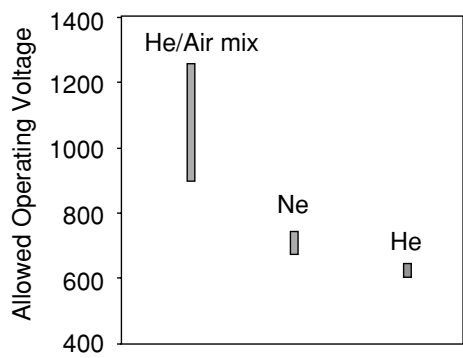

Fig. 14: Operational range of voltage on the microGeiger device for various fill gasses.

\section{CONCLUSIONS}

The microGeiger device was found to measure beta radiation produced from Uranium- $238{ }^{90} \mathrm{Sr},{ }^{60} \mathrm{Co}$, and ${ }^{204} \mathrm{Tl}$, all beta emitting isotopes. Counting rates of up to 24 counts per minute were measured, and were found to fall as the source moved away in distance with similarly to a laboratory grade Geiger counter. $\mathrm{He}$ and $\mathrm{Ne}$ were evaluated as background gasses, as they provide a reduced operating voltage. The voltage required for a He/Air mixture is higher, but it provides a larger range of biasing.

\section{ACKNOWLEDGEMENTS}

This work was supported primarily by the Engineering Research Centers Program of the National Science Foundation under Award Number EEC-9986866. The facilities used for this research include the Solid State Electronics Laboratory (SSEL) at the University of Michigan. The authors are grateful to Mr. Ramon Torres-Isea, for use of the Advanced Physics Laboratory. Travel support has been generously provided by the Transducers Research Foundation and by the DARPA MEMS and DARPA BioFlips programs.

\section{REFERENCES}

[1] S. A. Audet, E. M. Wouters, and M. H. Kim, "High-Purity Silicon Soft X-Ray Imaging Sensor", Sensors and Actuators, (A22) nos. 1-3, Mar. 1990 pp. $482-486$

[2] R. Wunstorf, "Radiation hardness of silicon detectors: current status", IEEE Transactions on Nuclear Science, (44) nos. 3, June 1997 pp. 806-14.

[3] E. Bertolucci, et. al., "BETAview: A Digital $\beta$-Imaging System for Dynamic Studies of Biological Phenomina," Nucl. Inst. and Methods, A381, 1996 pp. 527-530

[4] W. J. Price, Nuclear Radiation Detection, McGraw-Hill, 1964, pp. 123.

[5] G. Charpak, et. al., "Micromegas, a multipurpose gaseous detector," Nucl. Inst. and Methods, A478,2002 pp. 26-36

[6] G.F. Knoll, "Radiation Detection and Measurement," John Wiley and Sons, 2000, pp 161

[7] C.G. Wilson, Y.B. Gianchandani R.R. Arslanbekov, V. Kolobov, and A.E. Wendt, "Profiling and Modelling of DC Nitrogen Microplasmas," Jour. of Applied Physics, 94(5) Sept 2003, pp. 2845-51 